\title{
A BIG REMOTE SENSING DATA ANALYSIS USING DEEP LEARNING FRAMEWORK
}

\author{
Hanen Balti ${ }^{1}$, Imen Chebbi ${ }^{2}$, Nedra Mellouli ${ }^{2}$, Imed Riadh Farah ${ }^{1}$ and Myriam Lamolle ${ }^{2}$ \\ ${ }^{I}$ RIADI Laboratory, University of Manouba, Manouba, Tunisia \\ ${ }^{2}$ LIASD Laboratory, University of Paris 8 , Paris, France
}

\begin{abstract}
Spaceborne and airborne sensors deliver a huge number of Earth Observation Data every day. In this context, we can easily observe the whole earth from its different sides. Therefore, this big data is important in remote sensing and could be exploited in several domains requiring image classification, natural hazard monitoring, global climate change, agriculture, urban planning. Over the last five years, Convolutional Neural Networks (CNN) emerged as the most successful technique for the image classification task, as well as a number of other computer vision tasks. However, to train millions of parameters in CNN one requires a huge amount of annotated data. This requirement leads to a significant challenge if the available training data is limited for a target task at hand. To address this challenge, in the recent literature, researchers proposed various ways to apply a technique called Transfer Learning to transfer the knowledge gained by training CNNs parameters on some large annotated dataset to the target task with limited availability of training data. Most of our work in this paper was dedicated to proposing a hybrid classification of remote sensing images. This architecture combines Spark RDD image coding to consider image's local regions, pre-trained VGGNET-16 and UNET for image segmentation and SVM (Support Vector Machines) from spark Machine Learning to achieve labeling task.
\end{abstract}

\section{KEYWORDS}

Big Data, Remote Sensing, Deep Learning, Multi-label Classification, Feature extraction, Support Vector Machines

\section{INTRODUCTION}

Earth observation is a source of information in many fields of application such as remote sensing, cartography, aeronautics, etc. Over the years, the number of floating sensors in space is growing, hence the proliferation of captured images.

The various space borne and airborne sensors deliver a large number of earth observation data every day so that we can observe its different sides (Mingmin Ch et al., 2016). Indeed, these data are the main actor of big remote sensing data (BRSD) and has at least these classic 4Vs: the volume, the velocity, the veracity, and the variety.

Currently, the most active research area in machine learning (ML) is deep learning (DL). With its increased processing power and advances in processors and as the quantity of remote sensing data keeps rising, we are starting to talk about BRSD DL. It comes to play a major role in providing big remote sensing data analytic solutions for classification and clustering. Obviously, DL uses deep architectures in order to deal with complex relationships between the input data and the class label. In addition, DL and ensemble-based architectures are the most popular and efficient approaches for multi-source and multi-temporal land cover classification. They outperform traditional machine learning methods and cover both optical images and radar images. DL algorithms are showing better performance from hyper spectral and multispectral imagery such as extracting land cover types (Nataliia Kussu et al., 2017), pixel-based classification, semantic segmentation, or target recognition. They learn features from the data, where low-level features are extracted from texture and spectral information. The output features are represented at the highest level. In fact, DL has much well-established deep architecture like Deep Belief Network (DBN), Recurrent Neural Network (RNN), or Convolutional Neural Network (CNN). CNN is the most used architectures in image processing. This architecture is a multilayer network, composed of 2 stages: feature extractor and classifier. Many experiments have shown that 
the performance of remote sensing (RS) image scene classification has been significantly improved due to the powerful feature representation learned through different DL architectures (Xue-wen Chen, et al., 2014).

The most two devoted frameworks useful in deep learning are TensorFlow and Apache Spark. TensorFlow is an open source framework that is designated to ML and especially to DL. It has built-in support for DL and Neural Network (NN), so it makes easy to assemble a network, assign parameters and run the training process. Also, Tensorflow has a collection of samples trainable mathematic functions that are useful for NN. Due to the large collection of flexible tools, TensorFlow is compatible with many variants of ML. In addition, TensorFlow (Goldsborough, 2016) uses both CPUs and GPUs for computing hence it makes the compile time faster. Apache Spark is also an open source parallel computing framework, which has the advantage of MapReduce. It delivers flexibility, scalability, and speed to meet the challenges of Big Data. Spark integrates two main libraries SQL for querying large structured data and MLlib involving main learning algorithms with statistical methods (Anand Gupta et al., 2017).Obviously, MLlib is Spark's open-source ML library which includes several efficient training functionalities. It also supports different languages and provides a high-level API that rich Spark's ecosystem and facilitate the development of ML pipelines (Xiangrui Meng et al., 2017).

The purpose of our work is to analyze and classify heterogeneous multi-source remote sensing data in a parallel distributed architecture using two CNN models for feature extraction and SVM for the labeling. We used four different datasets according to image acquisition, image resolution, and image encoding in the perspective to evaluate the improvement of these two architectures.

The rest of this paper is organized as follows. In section II, we introduce the related work. In section III we present the proposed approach. Our results are detailed in section IV. A brief conclusion with perspectives for future studies is presented in Section V.

\section{RELATED WORK}

The most common challenge of RS applications is RS image classification. In fact, RS images can have a similar appearance but it belongs to different classes. Indeed, in a few recent years, the DL approaches come as a solution to this challenge. Deep Learning (DL) is proving that it has efficient results in hyperspectral and multispectral BRSD imagery in land cover types such as extracting forests, buildings, roads.

DL is taking off in remote sensing and many papers have been released talking about multiple applications of deep learning in remote sensing.

As DL is a deep feature learning architecture, it can learn semantic discriminative features and reach better classification compared with mid-level approaches.

In (Nataliia Kussul et al., 2017)a multilevel deep learning architecture is proposed using multi temporal images acquired by Landsat- 8 and Sentinel-1A satellites. The proposed architecture is four-level architecture, including preprocessing (level 1), supervised classification (level 2), post-processing (level 3) and final geospatial analysis (level 4). 1-D and 2-D CNN architectures are proposed to explore spectral and spatial features. At the preprocessing level, Self-organizing Kohonen maps (SOMs) are chosen for optical image segmentation and restoration of missing data. SOMs are trained for each spectral band separately. The restoration of the missing values is done by substituting input sample missing components with neuron's weight coefficient. The restored pixels are lately masked. In the second level (Supervised Classification with CNN), two different CNN architectures are compared: 1-D CNN in which convolutions are in the spectral domain and 2-D CNN in which convolutions are in the spatial domain. These two architectures are composed of two convolutions layers, max pooling layer, and two fully connected layers. The two architectures use different train filters and a different number of neurons in the hidden layers. A combination of AdaGrad and RMSProp are used for moment estimation and it proved better performance in term of fast convergence comparing to gradient descent or stochastic gradient descent. In the last level, (Post processing and Geospatial Analysis) several filtering algorithms have been developed. They are based on the information quality of the input data and field boundaries providing a pixel-based classification map. Finally, data fusion allows interpretations of the classification methods. Another work was proposed by (Nguyen et al, 2013)in which CNN architecture is used for satellite image classification. They used three convolutional and two sampling layers on resized and transformed input images to gray. The detail is described in (Nguyen et al, 2013). In (Gong Cheng et al, 2018)features are used directly from the FCN (Fully Convolutional Network) as the classifier inputs. In contrast, (Yiping Duan et al, 2017)proposed an approach in which the CNN pooling layer is substituted with a 
wavelet constrained pooling layer. This layer is used in conjunction with Markov Random Field and super pixel in order to provide a segmentation map. In ( Jie Geng et al, 2015)Used deep convolutional autoencoders (DCAE) for extracting features and automatic classification on high-resolution single polarization TerraSAR-X images. The architectures of the DCAE contain a convolutional hand-crafted first layer, and a scale transformation hand-crafted second layer using correlated neighbor pixel. The other layers are trained with a stacked autoencoder (SAE). In fact, (Ma, X et al , 2016)proposed a classification approach based on three decision levels: the first level is a local decision. A hyperspectral image will be sampled and the test sample is based on its neighborhood by calculating the Euclidean distance. The second level is a global decision based on supervised classification. It calculates a Euclidean distance between the sample and the classes. The third level is a self-decision. It is based on the label class involving spectral and spatial features. The first two decisions are applied to unlabeled samples in the training set. After that, the deep network is trained on the new training set to extract features and a classification map is generated from the self-decision.

In all the above-mentioned works, the heterogeneity of the images as well as their scaling, are considered separately. The aim of our work is to propose an adaptive framework to 1) solve the problem of scaling up and processing massive data using Spark RDD image coding to consider the image's local regions; 2) provide a semantic and generic classification tool processing multi-source and multi-resolution data using the best CNN models in the segmentation task and Spark Machine Learning in semantic labeling task.

\section{PROPOSED APPROACH}

Big Remote Sensing Data (BRSD) represents a challenge for the DL. In fact, BD involves a large number of samples as inputs, large varieties of classes as outputs and huge large dimensionality as attributes. This will lead to high running time and complex models. For all these reasons algorithms with a distributed framework and parallelized machines are required. In fact, we propose an approach based on a parallel architecture in order to classify BRSD. The approach is composed of three steps: data splitting, data processing and finally data reducing as following in figure ( $c f$. Figure 1).

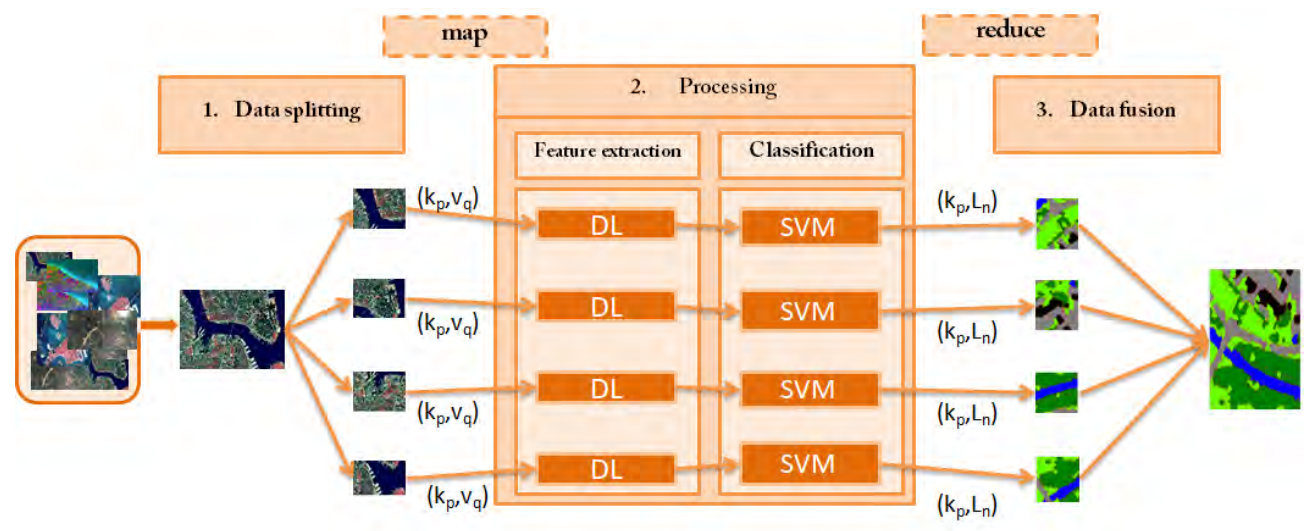

Figure 1. The proposed approach

First, we split the images into strips having the size of $144 * 144$ and distribute the data over several Spark-consumer nodes. Therefore, consistency between the outputs of this first step and the inputs of the next step must be maintained, since we have heterogeneous remote sensing images captured from several sensors like SPOT, LANDSAT, Worldview, etc. the fact that the resolutions and the number of bands differ from one image to another, like RGB (3 bands) or multispectral (more than 3 bands).

In fact, after splitting the data we obtain a number of images having a homogeneous resolution. This data are going to be stored in Resilient Distributed Datasets (RDD) using MapReduce technique in order to perform distributed and parallel processing on large datasets in a distributed environment, i.e. write parallel computations, using a set of high-level operators. In these RDDs, each image is considered as a dataset element. Hence each image is represented by a vector of RDDs records reflecting a local region of the image source. When the RDDs vectors are established, they are forwarded to the deep neural network inputs wherein the 
number of networks is equal to each vector dimension. At the second step, we are looking for feature extraction using CNN models. At the third step, we pipeline outputs of the last step to an SVM machine learning with MLlib package to aggregate local labels into a final image label classes.

\subsection{Remote Sensing Data Splitting and Storing in the RDDs}

As we have heterogeneous RS data; multi-resolution i.e. the images have different resolutions, multi-spectral i.e. the images can contain several bands such as Red, Green, Blue, Coastal, NIR (Near-infrared), SWIR (Short wavelet infrared), etc. we divided these images into strips having the size of $144 * 144$ i.e. we will deal with $n$ image each image have the size of $144 * 144$ and using the Map function each strip is processed to produce key-value pairs $\left(\mathrm{k}_{\mathrm{p}}, \mathrm{v}_{\mathrm{q}}\right)$ as intermediate outputs (Figure 2). On the value of these pairs, we are going to apply the processing step which includes two stages: Feature extraction and classification.

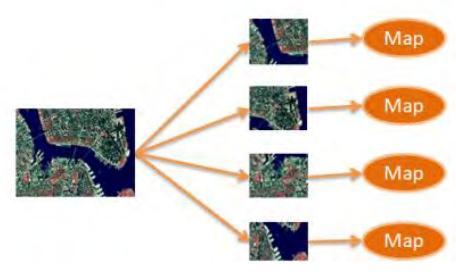

Figure 2. Image splitting task

The choice of the strip size was chosen by a heuristic approach taking into account the average size of an object to be detected based as well as all the resolutions of the original images. The detail of the splitting algorithm will be detailed in another paper.

For the storage and access of these strips, we decided to use RDDs because the only way to reuse our data between computations (Ex - between two "jobs" MapReduce) is to write them on an external stable storage system (Ex-HDFS). We used the concept of spark RDD to achieve faster and more efficient MapReduce operations. The RDDs are an immutable collection of distributed objects. Each set of data in RDD is divided into logical partitions, which are calculated on different nodes of the cluster. In addition, RDDs contain any type of Python object, Java or Scala, including the classes we have defined. The number of nodes that are in our cluster is the round of $(x * y / 144 * 144)$ where $\mathrm{x}^{*} \mathrm{y}$ is the image size and $144 * 144$ is the strip size.

The strips of images are stored as objects with their characteristics (origin, height, width, nChannels, mode, and data). After storing the images in these datasets we are going to apply the second step which is data processing.

\subsection{Remote Sensing Data Processing}

This step is composed of two sub-steps, as we mentioned above, feature extraction and classification.

\subsubsection{Feature Extraction}

Convolution is an efficient way to extract features (Han Liu, 2018), therefore, CNN architectures are suited for this task. In fact, we chose two CNN models, UNET and VGGNET, which are both of them, a stack of convolutional layers with pooling layers that are much like a filter to be applied to feature maps.

CNN architectures (but not limited to), UNET and VGGNET, to compare their added value in the face of image heterogeneity.

Feature extraction using UNET: A UNET is like a convolutional auto-encoder. It also has connections similar to the feature maps located before the bottleneck layer. It is generally used for semantic segmentation, in our case; UNET will be used as a feature extractor.

Firstly, we trained the model with 90000 strips of multispectral images, then as the first part of the model is composed of a stack of traditional convolutional layers, we take the features extracted from the bottleneck layer, i.e. the feature map extracted from the bottleneck layer is considered our output. 
Feature extraction using pre-trained VGGNET-16: The network is composed of 22 layers, 16 layers with learnable weights ( 13 convolutional layers, and 3 fully connected layers). For feature extraction, we neglected the last layer SOFTMAX layer, so the features are extracted from the last fully connected layer instead of the SOFTMAX layer.

Once the features are extracted, they will be stored locally in the RDDs in order to use them in the next task. Now, after getting the features extracted from the two models we are going to classify our images.

\subsubsection{Classification}

For this step, we used Linear Support Vector Machines (Linear SVM). Linear SVMs are supervised learning models with associated learning algorithms that analyze the data used for classification and regression analysis. The goal of the SVM algorithm is to find a hyperplane in $\mathrm{N}$-dimensional space (N - the number of features) that distinctly ranks the data points. In our work, the purpose of using SVMs is to find a plan that has the maximum margin, that is, the maximum distance between the data points of the two classes. Maximizing margin distance provides reinforcement for classifying future data points with greater confidence.

In order to adapt SVM to our work, we used the one against one approach which is a series of classifiers applied on each pair of class. Thus, $\mathrm{n}(\mathrm{n}-1) / 2$ machines are applied to each pair of classes. When all the classifiers have been attempted, a max-wins strategy has been used. The hyper-parameters that we used are the regularization parameter (or parameter C) and gamma. The Regularization parameter informs the SVM optimization how much we want to prevent misclassifying each training class and the gamma parameter describes how far the impact of a given example of training gets, with small values indicating "far" and elevated values indicating "near". In other words, with low gamma, points far from the plausible line of separation are regarded in the separation line calculation. Whereas in the calculation the points close to the plausible line are considered as high gamma. For the hyper-parameters selection, we used k-fold Cross Validation method.

The input of our algorithm is then the feature vectors of each strip of an image extracted with the UNET or VGGNET and stored locally in the RDDs. The output then is a $144 * 144$ image segmented and classified. Now, as we have the strips of images classified we will combine it in order to reconstruct the images.

\subsection{Remote Sensing Data Fusion}

After extracting the characteristics and classifying the images, we will now reconstructit. To perform this step, we will use the Reduce function of MapReduce. The reducer receives the key-value pair of the last several tasks, and then it includes the intermediate data tuples in a set of tuples, which are the final result. More specifically, the key-value pairs that our reduce will receive are the pairs $\left(\mathrm{k}_{\mathrm{p}}, \mathrm{L}_{\mathrm{n}}\right)$ with $\mathrm{L}_{\mathrm{n}}$ are the outputs of the classification step, that is, the classified strips of images are going to be grouped in order to reconstruct our images.

\section{EXPERIMENTAL RESULTS}

In this section, we will present our experimental setup, a description of data and the main results.

\subsection{Experimental Setup}

For the validation of our work, we created a machine on Amazon AWS having the following characteristics: $16 \mathrm{GiB}$ NVIDIA K80 GPUs, $64 \mathrm{vCPUs}$ and $61 \mathrm{GiB}$ of RAM, this machine is the master machine and the slaves have $8 \mathrm{GiB}$ Nvidia 950m GTX GeForce GPUs, they have quad-core CPU and $16 \mathrm{GiB}$ of RAM.

Data description: We have used four datasets to improve our approach: SIRI-WHU (Gong Cheng et al, 2017)which contains 12 classes each class contains 200 images measuring $200 * 200$ pixels with 2-m spatial resolution, AID (Gui-Song Xia et al, 2016)which contains 10000 images having several resolutions within 30 classes, multispectral dataset, and we prepared a dataset which is composed of satellite images captured with different sensors like SPOT4, SPOT5, LANDSAT8 and so on. Our dataset has a number of 3,974 images within 10 classes. The images have a various resolution, and the multispectral dataset contains $1 \mathrm{~km} \mathrm{x} 1 \mathrm{~km}$ satellite images in both 3-band and 16-band formats. The 3-band images are the traditional RGB natural color 
images. The 16-band images contain spectral information by capturing wider wavelength channels. This multi-band imagery is taken from the multispectral (400-1040nm) and short-wave infrared (SWIR) (1195-2365nm) range.

Evaluation metrics: The metrics that we used in order to evaluate the performance of our work are precision, recall, and F1-score.

$$
\text { Accuracy }=\frac{T P}{T P+F P} \quad \text { recall }=\frac{T P}{T P+F n} \quad \text { F1-score }=\frac{2 * \text { Accuracy } * \text { recall }}{\text { Accuracy }+ \text { recall }}
$$

where TP (True Positives) denotes the number of correctly detected objects, FN (False Negatives) the number of non-detected objects and FP (False positives) the number of incorrectly detected objects.

\subsection{Results}

In this part, we are going to present the results that we obtained after applying our approach on the BRSD. The experiments have been performed over two types of images: 3 bands (RGB) and 16 bands. Firstly, we should mention that we used $80 \%$ of our data for the training and $20 \%$ of the data for the validation. For the CNN models i.e. VGGNET and UNET we had 15000 iterations, the batch size was 128 and the learning rate was 0.001 .

In the following Table 1, we present the results obtained for each database using the 3 models; UNET, UNET-SVM and VGGNET-SVM.

Table 1. Comparison between the results obtained after testing our approach on 3 band databases and 16-band databases

\begin{tabular}{ccccccccccccc}
\hline & \multicolumn{3}{c}{ SIRI-WHU } & \multicolumn{3}{c}{ AID } & \multicolumn{3}{c}{ Our database } & \multicolumn{2}{c}{ 16- band database } \\
\hline & Accuracy & Recall & $\begin{array}{c}\text { F1- } \\
\text { score }\end{array}$ & Accuracy & Recall & F1-score & Accuracy & Recall & $\begin{array}{c}\text { F1- } \\
\text { score }\end{array}$ & $\begin{array}{c}\text { Accuracy } \\
\text { Rappel } \\
\text { score }\end{array}$ \\
\hline UNET & - & - & - & - & - & - & - & - & - & 0.92 & 0.57 & 0.70 \\
\hline UNET-SVM & 0.73 & 0.72 & 0.72 & 0.78 & 0.78 & 0.78 & 0.84 & 0.82 & 0.82 & 0.94 & 0.92 & 0.92 \\
\hline VGGNET-SVM & 0.73 & 0.68 & 0.70 & 0.67 & 0.50 & 0.55 & 0.79 & 0.80 & 0.79 & - & - & - \\
\hline
\end{tabular}

In the following Table 2, we present a comparison between the accuracy results obtained for our dataset per class using VGGNET-SVM and UNET-SVM.

Table 2. Comparison between the accuracy obtained for each class of our database using VGGNET-SVM and UNET-SVM

\begin{tabular}{lcc}
\hline Classes & VGGNET-SVM & UNET-SVM \\
\hline Buildings & 0.33 & 0.62 \\
\hline Cars & 0.61 & 0.69 \\
\hline Crops & 0.62 & 0.84 \\
\hline FastH2O & 0.55 & 1.00 \\
\hline Roads & 0.71 & 0.93 \\
\hline SlowH2O & 0.51 & 0.95 \\
\hline Structure & 0.57 & 0.82 \\
\hline Tracks & 0.42 & 0.75 \\
\hline Trees & 0.12 & 0.75 \\
\hline Trucks & 0.73 & 0.87 \\
\hline
\end{tabular}

In the following Table 3, we present a comparison between the accuracy results obtained in the state of art over the 3 bands datasets and our results

Table 3. Comparison between our results and the state-of-art results

\begin{tabular}{lc}
\hline & Overall precision \\
\hline (Gong Cheng et al, 2018) & $81 \%$ \\
\hline (Nataliia Kussu et al., 2017) & $93.6 \%$ \\
\hline (Nguyen et al, 2013) & $80.83 \%$ \\
\hline Our approach & $\mathbf{8 7 . 3 3 \%}$ \\
\hline
\end{tabular}


The execution time is very important since we deal with a huge and complex type of data and the fact of using a multi-node architecture helps us to gain in terms of time execution also. As a result, the mean execution time of our algorithm is 9.8 minutes.

\subsection{Discussion}

After presenting all the results in the previous section, we can say that using the encoder part of UNET as feature extractor is more efficient than VGGNET. In fact, the reshaping of pre-trained VGGNET can cause loss of image information. In contrast, the UNET model accepts any image with any size. Also, VGGNET is mainly used for RGB images contrariwise to UNET which don't impose RGB image as input, that's why we tested the UNET on the multispectral image (more than 3 spectral bands). We compared the results obtained with a simple UNET and the combination of UNET-SVM, the use of Linear SVM as classifier ameliorates the results. So the use of UNET allows us to gain in terms of precision also it allows us to manipulate data of different spectral resolution unlike the VGGNET with imposes the use of RGB images. In addition, the use of the distributed architecture allows us to manipulate images having different spatial resolutions i.e. to handle heterogeneous data. Our approach had slightly higher accuracy than (Gong Cheng et al, 2018) and (Nguyen et al, 2013) which used respectively FCN and a customized CNN architecture. In contrast, (Nataliia Kussu et al., 2017) with their multilevel deep learning architecture, outperform our approach results and this can be due to the first level in which they use SOMs for missing data restoration and optical image segmentation.

\section{CONCLUSION}

Big data and DL are both considered as a BIG deal for researchers. The concept of DL is to dig into a massive volume in order to identify patterns and extract features from complex unsupervised data without human intervention, which makes it an important tool for Big Data. In this paper, we proposed a DL algorithm for feature extraction and classifying satellite images based on two frameworks SPARK for storing, distributing and parallelizing Big Remote Data and Tensorflow for implementing and executing DL algorithms based on two CNN models (VGGNET16 and UNET). A benchmark dataset of remote sensing images was created for evaluation. For the future work, we are going to use hyperspectral Dataset for UNET results validation and we are going to develop other DL architectures in order to adapt our approach to different RS data types.

\section{REFERENCES}

Jie Geng et al. (2015). High-Resolution SAR Image Classification via Deep Convolutional Autoencoders. IEEE Geosci. Remote Sensing Lett. 12(11), 2351-2355.

Gong Cheng et al. (2018). When Deep Learning Meets Metric Learning: Remote Sensing Image Scene Classification via Learning Discriminative CNNs. IEEE Trans. Geoscience and Remote Sensing 56(5): , 2811-2821.

Han Liu, Y. (2018, 09). Feature Extraction and Image Recognition with Convolutional Neural Networks. Journal of Physics: Conference Series, 062032.

$\mathrm{Ma}$, X et al . (2016). Semisupervised classification for hyperspectral image based on multi-decision labeling and deep feature learning. ISPRS Journal of Photogrammetry and Remote Sensing, , 99-107.

Nataliia Kussul et al. (2017). Deep Learning Classification of Land Cover and Crop Types Using Remote Sensing Data. IEEE Geosci. Remote Sensing Lett. 14(5), 778-782.

Xiangrui Meng et al. (2017). A Big Data Analysis Framework Using Apache Spark and Deep Learning. Journal of Machine Learning Research 17, 9-16.

Xue-wen Chen, et al. (2014). Big Data Deep Learning: Challenges and Perspectives. IEEE Access 2 , 514-524.

Yiping Duan et al. (2017). SAR Image segmentation based on convolutional-wavelet neural network and markov random field. Pattern Recognition, 255-267.

Anand Gupta et al. (2017). A Big Data Analysis Framework Using Apache Spark and Deep Learning. ICDM Workshop, (pp. 9-16).

Goldsborough, P. (2016). A Tour of TensorFlow. CoRR abs/1610.01178. 
ISBN: 978-989-8533-92-0 @ 2019

Gong Cheng et al. (2017). Remote Sensing Image Scene Classification:Benchmark and State of the Art. Proceedings of the IEEE 105(10): , (pp. 1865-1883).

Gui-Song Xia et al. (2016). AID: A Benchmark Dataset for Performance Evaluation of Aerial Scene Classification. Proceedings of the IEEE 105(10): , 1865-1883.

Mingmin Ch et al. (2016). Big Data for Remote Sensing: Challenges and Opportunities. Proceedings of the IEEE, 104(11), 2207-2219.

Nguyen et al. (2013). Satellite image classification using convolutional learning. AIP Conference Proceedings (pp. 2237-2240). AIP. 